confusion were encountered more frequently in the $\mathrm{COHb}$ greater than $10 \%$ group compared with the group with $1,5 \%$ to $10 \%$ COHb levels.

Conclusions In this study, we managed to demonstrate the presence of more frequent symptoms in patients with a $\mathrm{COHb}$ level of $10 \%$ or greater. Further analysis revealed that severe symptoms were more pronounced in adolescents and that the severity of symptoms increased with age.

\section{PO-0308 DURAL SINUS AND INTERNAL JUGULAR VEIN THROMBOSIS AFTER BLUNT HEAD INJURY IN A PAEDIATRIC PATIENT: GOOD OUTCOME AFTER PROMPT ANTICOAGULATION THERAPY}

${ }^{1} P$ Berlese, ${ }^{1} G$ Casara, ${ }^{2} E$ Trincia, ${ }^{3} \mathrm{C}$ Costantini, ${ }^{3} \mathrm{~L}$ Grazian, ${ }^{4} E$ Nascimben, ${ }^{1} \mathrm{~L}$ Da Dalt. ${ }^{1}$ Department of Women and Child Health, University of Padua Pediatric Residency Program, Padua, Italy; ${ }^{2}$ Neuroradiology Unit, Treviso Hospital, Treviso, Italy; ${ }^{3}$ Pediatric Unit, Treviso Hospital, Treviso, Italy; ${ }^{4}$ Neurointensive Care Unit, Treviso Hospital, Treviso, Italy

\subsection{6/archdischild-2014-307384.958}

Introduction Paediatric cerebral venous sinus thrombosis (CVST) is a rare condition (3 cases/million population), usually idiopathic. Traumatic head injury is a rare cause of this severe disease and no consensus exists regarding diagnosis and management of post-traumatic CVST. We describe the case of a patient with blunt head injury who developed sigmoid sinus and Internal Jugular Vein Thrombosis (IJVT), successfully treated with anticoagulation therapy (ACT).

Case-Report A 14 years old boy, previously healthy, was brought to our ED because of an head trauma and transient loss of consciousness after a road traffic accident. On arrival he was conscious and irritated and needed sedation and oro-tracheal intubation. Immediate CT showed right temporo-parietal fracture with left fronto-parietal subarachoid hematoma. A 48 h CT revealed a worsening of right temporal hematoma and an hyperdensity area at the right sigmoid sinus suggestive of CVST, confirmed by CT venography as venous thrombosis of sigmoid sinus and IJV. His coagulation profile was normal. The patient started immediately Low Molecular Weight Heparin therapy, continued for three months. The MRI after two weeks of ACT was normal. He was discharged after 27 days without any neurological deficit. Conclusion Our report demonstrates importance of suspicion for CVST in head trauma, especially in those with high energy trauma or focal lesion, in which the neurological status cannot be monitored. The early diagnosis may permit to start an appropriated ACT, that is probably effective in reducing the risk of death and sequelae, if started early, during the acute phase.

\section{PO-0309 A BIRD'S EYE VIEW OF RETRIEVALS}

F Bickell, K McCulloch, K Starkie, E Hutton. Paediatric Intensive Care, Guy's and St Thomas' Foundation Trust, London, UK

\subsection{6/archdischild-2014-307384.959}

Background The increased use of simulation for teaching clinical and non technical skills has revolutionised our multidisciplinary retrieval training. The opportunity to experience real time situations and respond within a safe environment is widely documented as an invaluable teaching tool allowing teams to explore not only the treatments of different conditions but also to focus on the human factors and identify strategies for good leadership and follower ship, (Cheng et al 2007).
Method The extra benefit of the simulation days has come from an unexpected quarter. The teams themselves are asked to play differing roles fulfilling the position of local hospital staff or a parent. These experiences have resulted in unanticipated insights into what it is like to be involved in a retrieval from 'the other side'.

Results Retrieval team members have responded saying, 'the child was really ill and it was such a relief when the team arrived', showing real insight into the experiences of the local staff. 'It gave me a bird's eye view of retrievals'. Members playing the role of parents often became emerged in their roles feeling close to tears at times when things were not going well. Other comments included, 'there were so many of them I felt left out and out of control'.

Conclusions These comments will be further explored and discussed with relation to how these experiences can be translated into our practice and improve the service we deliver to our critically ill children and their families.

\section{P0-0310 MODIFIED BERDEN SCORE FOR PAEDIATRIC BASIC LIFE SUPPORT EXAMINATION}

M Binkhorst, M Hogeveen, Y Benthem, E van de Pol, J Draaisma. Paediatrics, Radboud University Nijmegen Medical Centre, Nijmegen, Netherlands

\subsection{6/archdischild-2014-307384.960}

Background and aims Although appropriate guidelines for paediatric basic life support (pBLS) are available (European Resuscitation Council (ERC) Guidelines, 2010) and many health care professionals follow pBLS courses, a reliable and valid scoring tool for pBLS examination is still lacking. We endeavoured to develop such a scoring instrument.

Methods Based on the ERC algorithm for pBLS and the scoring system for BLS in adults developed by Berden et al. (Resuscitation, 1992), we composed a scoring tool with content validity. Consensus on this scoring tool was reached within an expert panel to endow it with face validity. We used our tool for the pBLS examinations of 187 medical interns that just completed a pBLS course. All examinees were scored on-site and on video. Eighty video-recorded examinations were scored a second time by the same researcher after several months. An independent researcher also scored these 80 videos. Finally, 20 skilled pBLS course instructors were scored with our tool.

Results Thirty examinations were excluded, because videos were of insufficient quality or interns declined consent. Correlation between on-site and video-based scores was low. Less interns passed their pBLS examination based on on-site scores. Scoring was most divergent regarding the items chin lift, signs of life, leaning and tidal volume. When looking at the videos, intraobserver and inter-observer reliability were good. The percentage of pass scores was considerably higher in pBLS course instructors than in interns.

Conclusions We developed a reliable and valid scoring tool for pBLS examination. Video-based rather than on-site scoring is recommended.

\section{PO-0311 CEREBRAL SINOVENOUS THROMBOSIS IN CHILDREN}

D Boumendil, MA Negadi, K Elhalimi, H Bouguetof, Z Mentouri. Faculty of Medecine of Oran, CHU Oran, Oran, Algeria

10.1136/archdischild-2014-307384.961 
Introduction Cerebral venous thrombosis (CVT) is uncommon with a variable clinical presentation.

Heparin is used for first-line treatment in association with symptomatic and etiologic management.

Methods A retrospective review of the medical and radiological records of patients with CVT was managed in the Paediatric Intensive Care Unit, Faculty of Medicine- Oran University, Algeria over a six-year period.

We attempt to determine the clinical, radiological, etiologic and prognostic characteristics of CVT in this age range.

Results From 2008 to 2013, 05 children had cerebral venous thrombosis. Their ages ranged from 29 days to 10 years. Transient seizures (4 children), convulsive status epilepticus ( 1 patient) and focal neurologic signs (2 children) were the first clinical symptoms.

The presence of longitudinal (3 patients) and lateral (2 patients) sinus thrombosis was suggested by unenhanced CT scan, confirmed by magnetic resonance angiography, with absence of blood flow in the longitudinal sinus,

Thrombosis was associated with predisposing factors in all cases. Numerous causes or predisposing factors were identified: infections in 4 patients and non infectious causes in 3 patients.

Treatment consisted of anticoagulation in all cases, followed by oral anticoagulation.

The general outcome was good; in fact we observed a complete recovery.

Conclusion Diagnosis of sinus thrombosis can be made more accurately and noninvasively by angio-MRI.

\section{PO-0312 REFRACTORY CONVULSIVE STATUS EPILEPTICUS (RCSE) IN CHILDREN: CAUSES, RISK FACTORS AND OUTCOME}

D Boumendil, MA Negadi, K Elhalimi, H Bouguetof, Z Mentouri. Faculty of Medecine of Oran, CHU Oran, Oran, Algeria

\subsection{6/archdischild-2014-307384.962}

Introduction RCSE is a life-threatening neurologic emergency with high mortality and morbidity, in which seizures do not respond to first and second - line anticonvulsant drug therapy.

The aim of this study was to determine the risk factors, and the impact on the outcome of RCSE in children.

Patients and methods In this prospective study we analyse 53 children with RCSE.

Factors associated with seizure refractoriness were analysed using logistic regression.

Results Of 245 patients with convulsive status epilepticus, 53 developed RCSE (21.6\%), 37 of whom were males (70\%), with a mean age of 3 years ( 1 month-15 years). 11 patients (21\%) had a history of epilepsy.

The most frequent etiologies' were acute symptomatic (73, $6 \%$ ). Encephalitis 13 patients (24, 5\%), meningitis 8 patients (15\%), septic shock 5 patients $(9,4 \%)$, were the main acute etiologies.

Acute symptomatic causes (odds ratio [OR] 9, 95\% confidence interval $[\mathrm{CI}] 3.86-24.168 ; \mathrm{p}<1^{-4}$ ), duration of CSE $\geq 6$ h (OR 5,8\% [CI] 1.828-18.778; pp = 00034), complications (OR 13 [CI] 4.324-40.108; pp $<1^{-4}$ ) and received $>2$ doses of benzodiazepines (OR 5 [CI] 1.133-25.425; pp = 0034) were identified as independent risk factors for RCSE.

During hospitalisation 24 patients $(45 \%)$ died $\left(\mathrm{p}<1^{-4}\right)$. On discharge from hospital, 14 patients $(26.4 \%)$ presented new neurological deficit or difficult-to-manage epilepsy.
RCSE was associated with prolonged hospital length of stay ( $>3$ days) $(\mathrm{p}<1-4)$.

Conclusion GCSE termination and outcome seem clearly associated with adherence to treatment protocol, etiologies and duration of the CSE.

\section{PO-0313 FRENCH PAEDIATRIC INTENSIVE CARE UNITS POLICIES REGARDING ADMISSIONS OF ADOLESCENTS}

D Brossier ${ }^{1},{ }^{2} \mathrm{~N}$ Roumeliotis, ${ }^{1} \mathrm{~F}$ Villedieu, ${ }^{1} \mathrm{~N}$ Letouzé, ${ }^{1} \mathrm{~N}$ Pinto Da Costa, ${ }^{1} \mathrm{M}$ Jokic. ${ }^{1}$ Intensive Care Unit, CHU de Caen, Caen, France, ${ }^{2}$ Intensive Care Unit, CHU Sainte Justine, Montréal, Canada

\subsection{6/archdischild-2014-307384.963}

Background and aims In France, the department of paediatrics is expected to admit every patient up to 18 years. However, admission age is occasionally limited to 16 , even 15 , without any medical reason. In our opinion, patient overload is increasing in adult intensive care units (ICU), therefore the admission of adolescents should be PICU responsibility. We conducted a study evaluating PICU policies toward patients over the age of 15 years.

Methods A survey was conducted from July 25th to December 31st 2012. The PICUs listed in the GFRUP (Groupe Francophone de Réanimation et d'Urgence Pédiatrique) were asked to complete an on-line form about their unit, training level of the physicians and their unit policies concerning age limits for admission.

Results 22 units completed the form (55\%). Each PICU team was composed of at least one paediatrician and one physician trained in intensive care. $46 \%$ of PICUs occasionally admitted patients over 18 years of age. $96 \%$ admitted patients up-to 18 years, however among them only 33\% consistently admitted them without restriction. One unit admitted to never admitting patients over 15,25 years old, considering this age as the limit of paediatric practice.

Conclusion The PICUs were all equipped with adequately trained physicians to care for paediatric patients. The vast majority of PICUs admitted patients up to 18 years of age, but only a few considered this standard of care. They assume responsibility for problems related to long standing paediatric medical background or classic paediatric condition, regardless of patient age.

\section{PO-0314 ADMISSION OF PAEDIATRIC PATIENTS TO ADULT INTENSIVE CARE UNITS IN NORMANDY FROM 2008 TO 2010}

${ }^{1} \mathrm{D}$ Brossier, ${ }^{1} \mathrm{M}$ Jokic, ${ }^{2} \mathrm{~N}$ Roumeliotis, ${ }^{3} \mathrm{~B}$ Guillois. ${ }^{1}$ Intensive Care Unit, CHU de Caen, Caen, France; 'Intensive Care Unit, CHU Sainte Justine, Montréal, Canada; ${ }^{3}$ Neonatology, CHU de Caen, Caen, France

\subsection{6/archdischild-2014-307384.964}

Background and aims Paediatric intensive care units (PICU) are expected to admit every paediatric patient (age $<18$ years) with organ failure. Nevertheless, some of these patients are hospitalised in an adult ICUs (AICU). The purpose of this study was to evaluate and compare the paediatric patients admitted to AICUs in Normandy with those admitted to PICU.

Methods A retrospective study was conducted from January 1st 2008 to December $31 \mathrm{~s}^{\mathrm{t}} 2010$. Every paediatric patient admitted to an ACIU in Normandy was included (AICU group). The AICU group was compared to the paediatric patients admitted to 